\title{
SLIGHTLY MODIFIED TECHNIQUE OF THE ORIGINAL ESSED PLICATION PROCEDURE FOR CONGENITAL PENILE DEVIATION
}

\author{
C. VAN DER HORST ${ }^{(1)}$, F.J. MARTINEZ PORTILLO ${ }^{(1)}$, C. SEIF $^{(1)}$, A. MUSIAL ${ }^{(2)}$, P. ALKEN ${ }^{(2)}$, \\ K.P. JÜNEMANN ${ }^{(1)}$ \\ Department of Urology, University Hospitals of Kiel ${ }^{(1)}$ and Mannheim ${ }^{(2)}$, Germany
}

\begin{abstract}
Purpose: The Schroeder-Essed plication procedure is a standard technique for the correction of penile curvature. In a retrospective analysis we compared functional results and quality of life (LQ) of the original technique with inverted sutures as described by Schroeder-Essed and our slight modification consisting of horizontal incisions into the tunica albuginea.

Materials and Methods: Twenty-six patients with congenital penis deviation were treated for penile deviation by the original Schroeder-Essed plication with inverted sutures (11 patients) and by the described modification (15 patients). In case of modified technique, horizontal and parallel incisions $4 \mathrm{~mm}$ to $6 \mathrm{~mm}$ apart and about $8 \mathrm{~mm}-10 \mathrm{~mm}$ long were made through the tunica albuginea. The outer edges of the incisions were then approximated with permanent inverted sutures (Gore-Tex ${ }^{\circledR} 3$ 0 ). Mean age was 21.6 years in the first group and 23.2 years in the second group. Average follow-up was 28 months and 13 months, respectively. The preoperative penile deviation angle was $>25^{\circ}$ in all patients without difference between the 2 groups.

Results: All patients in both groups reported an improvement in their quality of life and full ability to engage in sexual intercourse. Nine patients (88\%) in the first group and 14 patients (93\%) in the second group were satisfied with the cosmetic result. In contrast, 10 patients $(91 \%)$ of the first and 13 patients $(87 \%)$ of the second group complained of penile shorting. Recurrence of deviation was only noticed in 2 males in the first group (18\%).

Conclusions: Our results indicate that this simple modification of the Schroeder-Essed plication offers good functional and cosmetic results. Most patients were satisfied with the penile angle correction results.
\end{abstract}

Key words: penis; congenital defect; curvature; tunica; reconstructive surgical procedures Int Braz J Urol. 2003; 29: 332-5

\section{INTRODUCTION}

Independent of its etiology, penile deviation may disturb sexual intercourse by difficult vaginal intromission. A simple standard method of congenital curvature repair is the Schroeder-Essed plication procedure (1). We describe and examine a slight modification of this technique consisting of horizontal incisions into the tunica albuginea avoiding cavernous tissue damage and improving adhesion of plicated tunical layers as similar performed by Baskin \& Duckett (2). The 2 techniques were analyzed retrospectively by comparing the functional results and life quality (LQ) using standardized questionnaires on their quality of life.

\section{MATERIALS AND METHODS}

Between June 1996 and July 2000, 26 patients with congenital penile deviation underwent the origi- 
nal Schroeder-Essed plication with inverted sutures (11 patients) and the described modification (15 patients). Mean age was 21.6 yrs in the first group and $23.2 \mathrm{yrs}$ in the second group. Average follow-up was 28 months and 13 months, respectively. The preoperative penile deviation angle was $>25^{\circ}$ in all patients without differentiation between the 2 groups, documented by means of auto-photography. Detailed information about possible post-operative discomforts or pain from suture knots, initial irregularities of the penile shaft and shortening of the penis was given to the patients preoperatively. A standardized questionnaire was sent to all patients. Retrospectively we examined quality of life, sexual intercourse, penile deviation, penile shortening, penile sensation, quality of erection, cosmetic result and recurrence.

\section{SURGICAL TECHNIQUE}

Surgery was performed under general anesthesia. A tourniquet was set at the base of the penis. An artificial erection was induced by injection of sterile saline solution into the corpora cavernosa through a $19 \mathrm{G}$ butterfly needle to determine the degree of deviation. The outer preputial layer is circumferentially incised at the level of the coronal sulcus to facilitate the drop back of the penile shaft skin. The plain of dissection is kept just outside of Buck's fascia in order to preserve the vascular pedicle to the dorsal hooded foreskin. Buck's fascia is elevated on the convex side by avoiding the neurovascular bundle of damage. To straighten the penis, the effect of the tunical plication was simulated by using Allis clamps under careful protection of the dorsal neurovascular bundle and its branches. In case of the original Schroeder-Essed technique plication is made by inverted sutures (1) (Gore-Tex $\left.{ }^{\circledR} 3-0\right)$. In case of modified technique, 2 parallel horizontal incisions $4-6 \mathrm{~mm}$ apart and about $8-10 \mathrm{~mm}$ long are made through the tunica albuginea. The outer edges of the incisions are approximated with permanent inverted sutures (Gore-Tex ${ }^{\circledR} 3$-0) (Figure-1). A straight penis is confirmed by repeated intra-operative artificial erection. The overlying penile fascia was closed in layers, and a circular dressing with light pressure was applied for a maximum of 2 days.

\section{RESULTS}

All patients in both groups undergoing plication for congenital deviation of the penis reported an improvement in their quality of life and full ability to engage in sexual intercourse. The penile deviation was abolished completely in all patients $\left(<15^{\circ}\right)$. Nine patients $(88 \%)$ in the first group and 14 patients $(93 \%)$ of the second group were satisfied with the cosmetic result. No patients reported any changes in penile sensation or in the quality of erections. In contrast, 10 patients $(91 \%)$ of the first and 13 patients $(87 \%)$ of the second group complained of penile shorting. Recurrence of deviation was only observed in 2 males in the first group (18\%) (Table-1).

Table 1 - Comparison of Schroeder-Essed procedure and the modified technique.

\begin{tabular}{|c|c|c|c|c|}
\hline Original & CPD & & Modification & CPD \\
\hline $100 \%$ & 11 & Improvement of LQ & $100 \%$ & 15 \\
\hline $88 \%$ & 9 & Cosmetic result & $93 \%$ & 14 \\
\hline $100 \%$ & 11 & Possible cohabitation & $100 \%$ & 15 \\
\hline $91 \%$ & 10 & Penile shorting & $87 \%$ & 13 \\
\hline $18 \%$ & 2 & Deviation recurrences & $0 \%$ & 0 \\
\hline
\end{tabular}

$C P D=$ congenital penile deviation; $L Q=$ quality of life. 


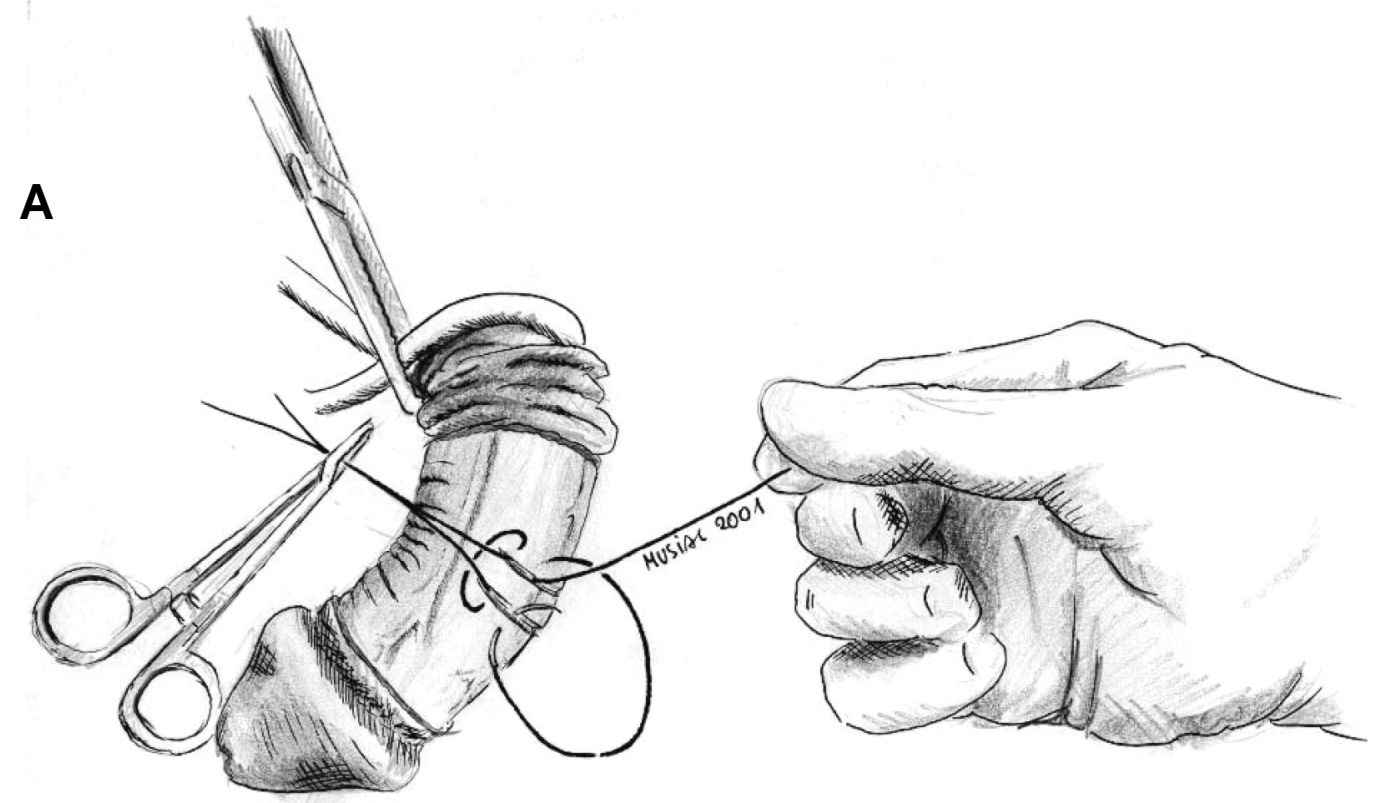

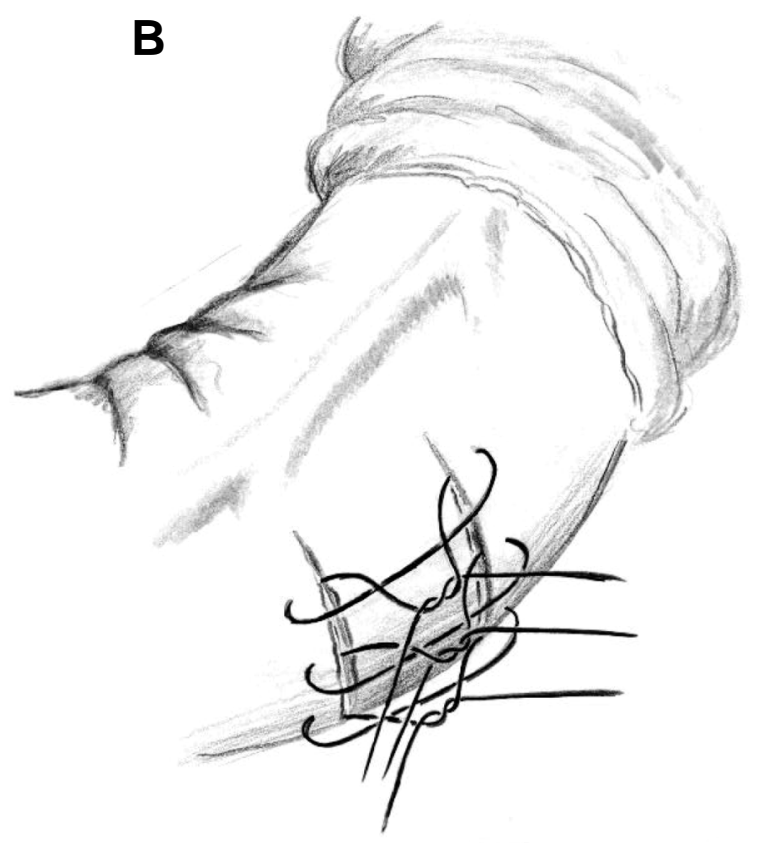

Figure - 1, A and B - Slightly modified technique of the original Essed plication procedure with 2 parallel horizontal incisions into the tunica albuginea.
Statistical analysis showed no significant difference between the 2 groups.

\section{COMMENT}

The basic surgical strategy for treating mean with disabling penile curvature and adequate erectile function entails lengthening the concave side of the curvature or shortening the convex side of the curvature. Whereas the first technique has the major problem of prolonged postoperative recovery time and possible subsequent erectile dysfunction $(3,4)$ the second technique leads to shortening of the penis. Nesbit described in 1965 the use of plication sutures in combination with removing elliptical segments of the tunica albuginea (5). Essed \& Schroeder plicated the tunica albuginea without incision (1). Our data show, that the incisions are less invasive in comparison with the removing of an elliptical segments of the tunica albuginea but may strengthen the plication by proper healing. We did not see any deviation recurrence or any changing in penile sensation. 


\section{CONCLUSIONS}

Our results indicate that this simple modification of the Schroeder-Essed plication can provide good functional and cosmetic results for patients with congenital penile deviation. The technique is simple, bloodless, safe and reliable. Most patients were satisfied with the penile angle correction results.

\section{REFERENCES}

1. Essed E, Schroeder FH: New surgical treatment for Peyronie disease. Urology 1985; 25: 582-7.
2. Baskin LS, Duckett JW: Dorsal tunica albuginea plication for hypospadias curvature. J Urol. 1994; 151: 1668-71.

3. Levine LA, Lenting EL: A surgical algorithm for the treatment of Peyronie's disease. J Urol. 1997; 158: 2149-52.

4. Jordan GH, Angermeier KW: Preoperative evaluation of erectile function with dynamic infusion cavernosometry/cavernosography in patients undergoing surgery for Peyronie's disease: correlation with postoperative results. J Urol. 1993; 150: 1138-42.

5. Nesbit RM: Congenital curvature of the phallus: Report of 3 cases with description of corrective operation. J Urol. 1965; 93 :230-2.

Received: December 27, 2002 Accepted: March 5, 2003

\section{Correspondence address:}

Dr. F. J. Martínez Portillo

Department of Urology

University Hospital Kiel

Christian-Albrechts-University Kiel

D-24105 Kiel, Germany

Fax: + 49621 597-1957

E-mail:fmartinez@urology.uni-kiel.de

\section{EDITORIAL COMMENT}

This retrospective study compares 2 cohorts of men with congenital penile deviation (chordee without hypospadias). The first group $(\mathrm{n}=11)$ had the original Schroeder-Essed plication using a technique described by Duckett for hypospadia repair. Average follow-up was 28 months for group 1 and 13 months for group 2.

The original Schroeder-Essed plication places non-absorbable sutures into the tunica albuginea in such a manner as to shorten the long axis of the penis when these sutures are tied. The problem with this type of plication is that there is no permanent remodeling of the tunica albuginea, and the curvature will recur if and when these sutures ever break. The modification involves making incisions in the tunica albuginea and securing then with sutures. Once healing takes place, even if the sutures break or are absorbed, the curvatures should not recur. The authors found no outcome differences in outcome between the 2 groups. The problem is that follow-up in each of the 2 groups is too short for the presumed benefit of the modification become evident.

Dr. Drogo Montague Glickman Urological Institute The Cleveland Clinic Foundation Cleveland, Ohio, USA 